Prof. Folie bespricht seine neueren Untersuchungen über die Veränderungen der Lage der Rotationsaxe der Erde in Bezug auf eine feste Ekliptik.

Dr. Schwarzschild hält einen Vortrag über die Frage, wie weit ein s.g. gekrümmter Raum angenommen werden könnte, ohne mit den astronomischen Beobachtungen in Widerspruch zu gerathen. Ausgehend von den Helmholtz'schen Vorstellungen zeigt er, dass unter Voraussetzung des pseudosphärischen Raums der Krümmungsradius > 4 Millionen Erdbahnradien angenommen werden müsse, während unter Voraussetzung des sphärischen Raumes der Krümmungsradius zwischen etwa 100 Mill. Erdbahnradien und unendlich als möglich zu erachten wäre.

Dr. Ebert spricht uber die von ihm in Gemeinschaft mit den Herren Renan und Perchot ausgeführte Untersuchung der Pariser Polhöhe vermittelst Beobachtung von polnahen Sternen am Gartenkreise der Pariser Sternwarte. Dr. Ristenpart schliesst hieran eine Mittheilung über eine äbnliche Aufgabe, die Prof. Harzer dem demnächst in Kiel -zu errichtenden grossen Meridiankreise zuzuweisen gedenkt.

Prof. Weiss macht die erfreuliche Mittheilung, dass ein Liebhaber der Astronomie, Herr Lindemann,-beabsichtige, zur Beschleunigung der rechnerischen Aufarbeitung des Cometenmaterials aus der älteren Zeit bis gegen die Mitte des 1 9. Jahrhunderts geeignete Geldmittel zur Verfügung zu stellen. Eine nähere Mittheilung hierüber wird demnächst an passender Stelle erfolgen.

Am 10. A ugust folgten die Mitglieder einer Einladung der grossh. Staatsregierung zu einem Ausfluge nach BadenBaden.

Die in der dritten Sitzung am I I. August zunächst vorgenommenen Wahlen ergaben die Wiederwahl der statutenmässig ausscheidenden Vorstandsmitglieder, sowie von Prof. Seeliger als Vorsitzenden. Als nächster Versammlungsort wurde Göttingen gewählt.

Prof. Förster berichtet über einige Aenderungen, die er in seinem in den Berl. Astr. Jahrb. 1880 und 1882 niedergelegten Verfahren tuber die Bestimmung absoluter Declinationen und absoluter Polhöhen vorgenommen hat.

Prof. Brendel und Prof. F. Klein berichten über die

Kiel, I 900 August.
Weiterführung der von der Göttinger Gesellschaft der Wissenschaften veranstalteten Gesammtausgabe von Gauss' Werken. Da ein Prospect hieruber demnächst in weiteren Kreisen verbreitet werden wird, sei hier nur erwähnt, dass der 7 . Band ausser einem Abdruck der Theoria motus die sämmtlichen Störungsrechnungen von Gauss enthalten wird. Die letzteren sind in vieler Beziehung von grossem Interesse; so z. B. hat Gauss von 1810 ab allgemeine Störungen in einer Form berechnet, welche der Hansen'schen Methode sehr ähnlich ist.

Prof. F. Klein berichtet über die Ausdehnung der Encyklopädie der mathematischen Wissenschaften auf die angewandte Mathematik, insbesondere auf die Astronomie.

Prof. Brendel legt einige Nordlichtphotographien vor. Dr. Hartzig macht auf die günstige Gelegenheit aufmerksam, aus dem Nachlasse des Uhrmachers Ort in Bamberg eine vorzügliche Pendeluhr zu erwerben.

Prof. Weiss berichtet über verschiedene an der Wiener Sternwarte unternommene Catalogisirungsarbeiten.

Prof. Ambronn legt Proben des Magnaliums, einer neuen Legirung von Aluminium und Magnesium, vor und spricht über deren Anwendung bei der Herstellung von wissenschaftlichen Instrumenten.

Prof. Charlier hält einen Vortrag über neue. Untersuchungen auf dem Gebiete der säcularen Störungen der kleinen Planeten.

Herr Witt spricht über die Prüfung eines nach den Schupmann'schen Ideen angefertigten Medialfernrohrs, die ein vorzügliches Resultat ergeben hat. Im Anschluss hieran berichtet Dr. Pauly uber von der Firma Zeiss unternommene Versuche, dieses Princip in die astronomische Beobachtungskunst einzuführen; ein grosses Bedenken sei, dass die geringste Störung der Orientirung ungünstig auf die Chromasie etc. einwirke.

Dr. Ristenpart spricht an der Hand der Publ. X der Kieler Sternwarte über die in Kiel befolgte Methode der Zeitbestimmung im Vertical des Polarsterns.

Die Versammlung schliesst mit dem lebhaften Dank gegenüber der grossh. Staatsregierung, der Heidelberger Stadtbehörde und den Heidelberger Astronomen für die erwiesene Gastfreundschaft.

\title{
Terza parte dell' effemeride di (433) Eros.
}

$\mathbf{I}^{\mathrm{h}}$ t. m. Berlino.

\begin{tabular}{|c|c|c|c|c|c|c|c|c|}
\hline \multicolumn{2}{|c|}{1901} & \multicolumn{2}{|r|}{$\alpha$ vera } & \multicolumn{3}{|c|}{$\delta$ vera } & $\log \Delta$ & t. di ab. \\
\hline Gen. & 1 & $\mathbf{2}^{\mathrm{h}}$ & $3^{m} 5^{2} .80$ & $+37^{\circ}$ & $55^{\prime}$ & I 4 " 5 & 9.499306 & $2^{\mathrm{m}} 3^{8^{\mathrm{s}}}$ \\
\hline & 2 & & $\begin{array}{ll}6 & 25.95\end{array}$ & 37 & 29 & 26.7 & 499666 & 38 \\
\hline & 3 & & $9 \quad 2.80$ & 37 & 3 & 39.5 & $50008 \mathrm{I}$ & $3^{8}$ \\
\hline & 4 & & 143.25 & 36 & 37 & 53.0 & 500551 & $3^{8}$ \\
\hline & 5 & & 427.17 & $3^{6}$ & 12 & $7 \cdot 3$ & 501078 & $3^{8}$ \\
\hline & 6 & & $17 \times 4.42$ & 35 & 46 & 22.6 & $50166 \mathrm{I}$ & $3^{8}$ \\
\hline & 7 & & $20 \quad 4.92$ & 35 & 20 & 39.0 & 502300 & 39 \\
\hline & 8 & & $22 \quad 5^{8.55}$ & 34 & 54 & 56.7 & 502995 & 39 \\
\hline & 9 & & $555.2 \mathrm{I}$ & 34 & 29 & 15.8 & $5 \circ 3747$ & 39 \\
\hline & 10 & & 854.80 & 34 & 3 & $3^{6.6}$ & 504557 & 40 \\
\hline & I I & 23 & 3157.23 & +33 & 37 & 59.2 & 9.505424 & 240 \\
\hline
\end{tabular}

\begin{tabular}{|c|c|c|c|c|c|c|c|}
\hline I 901 & \multicolumn{2}{|c|}{$\alpha$ vera } & \multicolumn{3}{|c|}{$\delta$ vera } & $\log 4$ & t. di ab. \\
\hline Gen. I 2 & $2^{\mathrm{h}} 35$ & $2 \cdot 39$ & $+33^{\circ}$ & 12 & 23.8 & 9.506347 & $2^{m} 40^{s}$ \\
\hline 13 & 38 & 10.17 & 32 & 46 & 50.5 & 507329 & $4 I$ \\
\hline 14 & $4 I$ & 20.46 & 32 & 21 & 19.6 & 508369 & $4 I$ \\
\hline 15 & 44 & 33.19 & $3 \mathrm{I}$ & 55 & 51.2 & $5 \circ 9467$ & $4 I$ \\
\hline 16 & 47 & 48.27 & $3^{I}$ & 30 & 25.6 & 510622 & 42 \\
\hline 17 & 51 & $5.6 \mathrm{I}$ & 31 & 5 & 3.0 & 511835 & 42 \\
\hline 18 & 54 & 25.10 & 30 & 39 & 43.4 & 513106 & 43 \\
\hline 19 & 257 & 46.65 & 30 & 14 & 27.1 & 514434 & 43 \\
\hline 20 & 31 & 10.19 & 29 & 49 & 14.4 & 515820 & 44 \\
\hline 21 & 4 & 35.62 & 29 & 24 & 5.4 & $51726 I$ & 44 \\
\hline 22 & 3 & 2.86 & +28 & 59 & 0.3 & 9.518758 & 245 \\
\hline
\end{tabular}




\begin{tabular}{|c|c|c|c|c|c|c|c|c|}
\hline \multirow{2}{*}{$\frac{1901}{\text { Gen. } 22}$} & \multicolumn{3}{|c|}{$\alpha$ vera } & \multicolumn{3}{|c|}{$\delta$ vera } & \multirow{2}{*}{$\frac{\log A}{9 \cdot 5^{1875^{8}}}$} & \multirow{2}{*}{$\frac{\text { t. di ab. }}{2^{m} 45^{s}}$} \\
\hline & $3^{h}$ & $8^{n}$ & n 2.86 & +28 & ${ }^{\circ} 59^{\prime}$ & 0.3 & & \\
\hline 23 & & II & 31.79 & 28 & 33 & $59 \cdot 3$ & 520310 & 45 \\
\hline 24 & & 15 & $2 \cdot 31$ & 28 & 9 & 2.5 & 521918 & 46 \\
\hline 25 & & 18 & $34 \cdot 34$ & 27 & 44 & I 0.1 & 523579 & 47 \\
\hline 26 & & 22 & 7.78 & 27 & 19 & 22.2 & 525294 & 47 \\
\hline 27 & & 25 & 42.53 & 26 & 54 & 39.0 & 527062 & 48 \\
\hline 28 & & 29 & 18.47 & 26 & 30 & 0.5 & 528884 & 49 \\
\hline 29 & & 32 & 55.50 & 26 & 5 & 26.8 & $53075^{8}$ & 49 \\
\hline 30 & & 36 & 33.53 & 25 & 40 & $5^{8.2}$ & 532685 & 50 \\
\hline 31 & & 40 & 12.48 & 25 & 16 & 34.8 & 534664 & 51 \\
\hline Febb. I & & 43 & 52.25 & 24 & 52 & 16.6 & 536695 & 51 \\
\hline 2 & & 47 & 32.76 & 24 & 28 & 3.8 & $53^{8779}$ & $5^{2}$ \\
\hline 3 & & 51 & 13.94 & 24 & 3 & $5^{6.7}$ & 540917 & 53 \\
\hline 4 & & 54 & 55.72 & 23 & 39 & 55.4 & 543106 & 54 \\
\hline 5 & 3 & $5^{8}$ & $3^{8.03}$ & 23 & 16 & 0.1 & 545347 & 55 \\
\hline 6 & 4 & 2 & 20.80 & 22 & $5^{2}$ & I 1.1 & 547640 & 56 \\
\hline 7 & & 6 & 3.97 & 22 & 28 & 28.6 & 549986 & 57 \\
\hline 8 & & 9 & 47.49 & 22 & 4 & 52.9 & $55^{2} 3^{8} 3$ & $5^{8}$ \\
\hline 9 & & 13 & $31.3^{\circ}$ & 21 & 41 & 24.2 & $554^{8} 31$ & $5^{8}$ \\
\hline 10 & & 17 & 15.37 & 2 I & 18 & 2.8 & $55733^{\circ}$ & 259 \\
\hline I I & & 20 & 59.64 & 20 & 54 & 48.9 & 559879 & 30 \\
\hline 12 & & 24 & 44.07 & 20 & 31 & 42.8 & 562477 & I \\
\hline 13 & & 28 & 28.61 & 20 & 8 & 44.7 & 565124 & 2 \\
\hline 14 & & 32 & 13.24 & 19 & 45 & 54.7 & 567818 & 4 \\
\hline 15 & & 35 & 57.91 & 19 & 23 & 13.0 & 570561 & 5 \\
\hline 16 & & 39 & 42.58 & I 9 & 0 & 39.8 & $9.57335^{\circ}$ & 37 \\
\hline 17 & & 43 & 27.24 & 18 & 38 & 15.7 & & \\
\hline 18 & & 47 & 11.84 & 18 & 16 & 0.5 & $9 \cdot 579062$ & 3 \\
\hline 19 & & 50 & 56.35 & 17 & 53 & 54.5 & & \\
\hline 20 & & 54 & 40.73 & 17 & 31 & 57.7 & $9 \cdot 5^{8} 4943$ & 312 \\
\hline 21 & 4 & $5^{8}$ & 24.94 & 17 & 10 & 10.4 & & \\
\hline 22 & 5 & 2 & 8.96 & 16 & 48 & 32.5 & 9.590984 & 314 \\
\hline 23 & & & 52.72 & 16 & 27 & $4 \cdot 3$ & & \\
\hline 24 & & 9 & 36.20 & 16 & 5 & 45.8 & $9.597 \times 75$ & $\begin{array}{ll}3 & 17\end{array}$ \\
\hline 25 & 5 & 13 & 19.36 & -15 & 44 & 37.2 & & \\
\hline
\end{tabular}

\begin{tabular}{|c|c|c|c|c|c|c|c|c|c|}
\hline \multirow{2}{*}{$\frac{1901}{\text { Febb.26 }}$} & \multicolumn{3}{|c|}{$\alpha$ vera } & \multicolumn{3}{|c|}{$d$ vera } & \multirow{2}{*}{$\frac{\log \Delta}{9.6035 \circ 7}$} & \multicolumn{2}{|c|}{ t. di $a b$. } \\
\hline & $5^{h} t$ & $\mathrm{~m}$ & 25.16 & $+15^{\circ}$ & ${ }^{\circ} 23^{\prime}$ & 38.4 & & & $\mathrm{~m}_{20} \mathrm{~s}$ \\
\hline & 2 & & 44.55 & 15 & 2 & 49.5 & & & \\
\hline & & & 26.51 & 14 & 42 & 10.7 & 9.609972 & 3 & 23 \\
\hline Mar. & & & 8.01 & 14 & 21 & 42.1 & & & \\
\hline & 3 & & 49.02 & 14 & 1 & $23 \cdot 7$ & 9.616564 & 3 & 26 \\
\hline & 3 & & $29.5^{\circ}$ & 13 & $4 r$ & 15.5 & & & \\
\hline & 3 & & 9.44 & I 3 & 21 & 17.7 & $9.6232 y 6$ & 3 & 29 \\
\hline & 4 & & 78.84 & 13 & I & 30.4 & & & \\
\hline & 4 & & 27.67 & 12 & 41 & 53.5 & 9.630104 & 3 & 33 \\
\hline & & & 5.92 & 12 & 22 & 27.1 & & & \\
\hline & 5 & & $43 \cdot 5^{8}$ & 12 & 3 & I I. 2 & 9.637041 & 3 & $3^{6}$ \\
\hline & 55 & & 0.65 & I I & 44 & 5.9 & & & \\
\hline & & & 77.11 & I I & 25 & II.I & 9.644083 & 3 & 40 \\
\hline & & & 32.97 & I 1 & 6 & 26.9 & & & \\
\hline & & & 8.23 & 10 & 47 & 53.2 & 9.651223 & 3 & 43 \\
\hline & $\mathbf{r}$ & & 72.89 & 10 & 29 & 29.9 & & & \\
\hline I & $\mathbf{t}$ & & 6.94 & 10 & I I & 17.1 & $9.65^{8455}$ & 3 & 47 \\
\hline & I & & 50.40 & 9 & 53 & 14.6 & & & \\
\hline I & 2 & & 3.26 & 9 & 35 & 22.4 & $9.665772^{\circ}$ & 3 & 51 \\
\hline & & & 5.53 & 9 & 17 & 40.3 & & & \\
\hline & 2 & & 7.22 & 9 & 0 & 8.4 & 9.673169 & 3 & 55 \\
\hline I & 3 & & 58.34 & 8 & 42 & 46.2 & & & \\
\hline & 3 & & 8.88 & 8 & 25 & 33.8 & $9.680635^{\circ}$ & 3 & 59 \\
\hline 2 & 3 & & $; 8.85$ & 8 & 8 & 31.0 & & & \\
\hline 2 & 4 & & 8.24 & 7 & $5^{I}$ & 37.6 & 9.688164 & 4 & 3 \\
\hline 2 & 4 & & $\$ 7.04$ & 7 & 34 & 53.4 & & & \\
\hline 2 & 5 & & 5.25 & 7 & 18 & 18.4 & 9.695746 & 4 & 7 \\
\hline & & & 2.85 & 7 & $\mathbf{I}$ & 52.3 & & & \\
\hline 2 & 65 & & 9.84 & 6 & 45 & 35.1 & $9.7 \circ 3374^{\circ}$ & 4 & 12 \\
\hline & 7 & & 6.21 & 6 & 29 & 26.6 & & & \\
\hline 2 & & & 1.95 & 6 & 13 & 26.7 & 9.711043 & 4 & 16 \\
\hline 2 & & & 37.07 & 5 & 57 & $35 \cdot 3$ & & & \\
\hline 3 & $\mathbf{I}$ & & $1.5^{6}$ & 5 & 41 & 52.2 & 9.718749 & 4 & 2 I \\
\hline 3 & & & 0 & 5 & 26 & $17 \cdot 3$ & & & \\
\hline Apr. & $7 i$ & & $8.6 \mathrm{I}$ & +5 & 10 & 50.5 & 9.726487 & & 26 \\
\hline
\end{tabular}

$\begin{array}{rcc}\text { 1901 } & \log r & \text { Gr. (oss.dir.) } \\ \text { Gen. } 3 & 0.065973 & 8.44 \\ 7 & 0.063565 & 8.45 \\ 11 & 0.061407 & 8.45 \\ 15 & 0.059512 & 8.46 \\ 19 & 0.057893 & 8.47 \\ 23 & 0.056562 & 8.49 \\ 27 & 0.055530 & 8.52 \\ 31 & 0.054802 & 8.55\end{array}$

\begin{tabular}{rcc}
\multicolumn{1}{c}{ 1901 } & $\log r$ & Gr. (oss.dir.) \\
Febb. 4 & 0.054386 & 8.6 \\
8 & 0.054283 & 8.6 \\
12 & 0.054495 & 8.7 \\
I6 & 0.055021 & 8.8 \\
20 & 0.055855 & 8.8 \\
24 & 0.056992 & 8.9 \\
28 & 0.058425 & 8.9 \\
Mar. 4 & 0.060141 & 9.0
\end{tabular}

$\begin{array}{rcc}\text { IgoI } & \log r & \text { Gr. (oss.dir.) } \\ \text { Mar. } 8 & 0.062129^{\circ} & 9.1 \\ 12 & 0.064377 & 9.2 \\ 16 & 0.066867 & 9.3 \\ 20 & 0.069586 & 9.3 \\ 24 & 0.072515 & 9.4 \\ 28 & 0.075639 & 9.5 \\ \text { Apr. I } & 0.078939 & 9.6\end{array}$

I luoghi geocentrici di Eros, da Igor febbrajo I6 a Igor aprile I, vennero calcolati, dietro mia preghiera, e per sua cortesia, dal Prof. Dr. A. Antoniazzi, astronomo del R. Osservatorio di Padova.

Roma, 1900 Agosto 30.

E. Millosevuch.

Planot (24) Thomis. Corr. der Ephemeride (B. J. 1902): Sept. 2 - $1^{8} 3^{6}-5.0$ Gr. 11.0. W. Luther.

Inhalt zu Nr. 3662. H. Osthoff. Ueber farbenwechselnde Fixsterne. 241. - Rambaud et Sy. Observations de planetes. 247. Bericht über die 18. Versammlung der Astronomischen Gesellschaft. 251. - E. Millosevich. Terza parte dell' effemeride di (433) Eros. 253. - W. Luther. Planet (24) Themis. 255. 